Le présumé contrefactour peut alors et suivant les cas, soutenir' une série de moyens contre l'exécution de l'ordomnance.

Il peut soutenir qu'il n'y a pas lieu à saisie, qui paralyse d'une façon inutile son exploitation et qu'une description est suffisante pour garantir les intérêts que veut défendre le breveté.

Il peut soutenir, en cas de contrefaçon de produit, qu'il esi inutile de saisir une série de produits et que la saisie d'un seul produit est suffisante pour permettre de rapporter, le cas échéant, la preuve de la contrefaçon.

Il peut soutenir que le breveté doit fournir un cautionnement préalablement à la saisie.

Il peut s'opposer à la saisie de la correspondance et des facturcs, soutenant qu'elle est illégale et qu'elle ne peut pas atre aulorisée par le Président du Tribunal dont l'ordonnance est, de ce chef, entachée d'excès de pouvoir.

Ces protestations sont-elles de nature à empêcher ou à suspendre les opérations?

Les ordonnances autorisant perquisition ou saisie, sont rendues en général à charge de référ er en cas de difficultés. l. breveté saisira le Président statuant en état de référé pour fance vider l'incident.

Les opérations seront suspendies en principe jusqu'à la solulion du référé, sauf dans le cas où l'ordonnance conliendra la formule suivante qu'il est prudent, pour celui qui poursuit la contrefaçon, de faire insérer : "Disons qu'en cas de difficultés, il nous en seria référé, mais après constatation préalablement faite ". Dans ce cas, les protestalions n'empêcheront pas l'exécution de l'ordonnance et, le nas ćchéant, la constatation de la contrefaçon.

(à suivre)

A. Bugand.

Arocat ì la Cour d'Appel de Lyon.

\section{ÉCONOMIE MONTAGNEUSE}

\section{UN PROBLÈME SOCIAL}

DANS LES MONTAGNES MÉRIDIONALES FRANGAISES (

Sur l'ensemble des régions françaises en proie à la dépòpulation contemporainc, le groupe de nos 3r départements montagneux du Midi est celui qui paie depuis longtemps le plus lourd tribut au flćau. La situation généralement très accidentée et l'onsemble des autres conditions géographiques de ces pays méridionaux y prédisposent le sol aux dégradations torrentielles; celte situation y impose le plus souvent la culture pastorale dont les abus provoquent toujours celte dégradation, surtout, comme c'est te cas, quand le mouton domine dans le capital pécoral ; enfin les conditions naturellement difficiles de la vic montagneuse y poussent les habitanls aux migrations, aux dépaysements. Il est donc légitime de chercher dans des contingences géographiques, ćconomiques et sociales, les éléments d'un Probleme social que ne sc sont pas explicitement posé jusqu'ici les restaurateurs du sol montagneux; et qui doit viser plus que jamais aujourd'hui la stabilisation et l'évolution progressive de nos montagnards. Discuter largement et attentivement ces questions est d'autant plus opportun qu'une législation

(1) Analyse d'un mémoire nrésenté par l'auteur à la a Section des sciences pronnminnes et sociales du 510 Congrés fles socibtés savantes o à Crenoble fe 13. mai 1913, pn rénonse à la deuxième queslion dn nrogramme: Rechercher dans une région les causes économiques de la desertion des campanneset les moyens suscoptibles d'en arretcr le mourement. montagneuse nouvelle dont les effels sociaux peuvent être désastreux, est actucllement pendante au Parlement.

Pour la masse de nos montagnards méridionaux, le dérncinement équivaut aujourd'hui à l'expatriation el le plus souvent à la dénationalisalion (1) : leur retour au pays est des plus problématiques. Des travailleurs étrangers se substituent aisément à eux, s"installent même souvent sur leurs " coins de terre " délaissés. Une des raisons matérielles et capilales de celte évasion se trouve dans la dégénérescence, en qualité et en quantité, des anciens communaux sur lesquels s'alimentait le bétail, matière de vie de ces populations pastorales. Les abus de l'élevage extensif inhérents en principe à la jouissance collective et qui ne peut y être autre, ont évincé bêtes et gens de paturages stérilisés et torrentialisés pour longtemps. Lcs énergies du pays se trouvent gravement atteintes par ce donble jeu d'évasion métropolitaine el d'invasion étrangère.

La colonisation officielle en Algéric a assumé une large part de responsabilité dans cette crise. C'cst elle qui orgatnisa l'exode il y a 30 ans quand, en suile de la législation montagneuse du 4 avril 188. , débuta la nationalisation des sols à restaurer ( $\stackrel{2}{)}$. L'Etat était alors bien décidé, quoique déjà pleinement conscient des dévastations matérielles, à limiter la restauration projetée aux seuls territoircs absolument dégradés, sans prendre, à l'égard des autres, aucune mosure préventive. Il a jusqu'à présent nationalisé, par expropriation ou acquisition amiable, plus de 200.000 hectares, en grande partie communaux. Les doléances des nombreuses fomilles qui les pouplaient, ct dont certaines comptaient plus de ro personnes, furent apaisées avec des lots de colonisation de terrains expropriés ou simplement confisqués aux Arabes. La colonisation officielle est aujourd'hui condamnée par tout le monde, même dans la petite patrie. Mais, en France, la nationalisation du sol fonctionne toujours, à raison de cinq à six mille heclares par an ; on la stimule, il faut bien dépenser les crédits ! C'est, au bas mot, une centaine de familles rurales de la grande patrie, que l'Etat voue ainsi et très chèrement, au prix de plus de 500 ooo fr., it la dénationalisation.

C'est à un pur mirage hydrologique que le pays est redevable de cet étrange et coûleux procédé de restauration monlagneuse... au moyen du vide ! Les lechniciens d'il y a cinquante ans, hantés par l'idée quo le roboisement des montagnes qu'on avait mis des siècles à dénuder sans que nul n'en prît cure, garantirait à jamais nos plaines d'inondation's désastreuses qui sont et resteront probablement dans le tempéramment de cortains grands fleuves, pour ne pas dire de tous, conduisirent le législateur, par une mésadaptation absolue de la loi de 18 ir sur les "grands travaux" d'utilité publique, et en vue d'une problématique sécurité à assurer aux gens de l'aval, à exproprier ceux de l'amont trop obstinés à refuser les futurs bienfaits d'un reboisement auxquels eux et leurs troupeaux avaient immédiatement fout ¿ perdre. Une expérience de trente ans réaliséc au prix do cent millions de francs, a mis en complète faillite celte restauration où les grands travaux architectoniques prirent une part démesurée ; clle a clairement montró les dangers sociaux provoqués par cette spéculation hydrologique qui, dans certaines régions très torrentielles, n'a guère eu du rboisement que lo nom.

(1) Lévasion contemporaine des montamards francals. (Annales de la science agronnmique française et btrangere. Janvier 1911,$0 ; 1-51)$.

Gestauration et nalionalisation du sol en hate montagne, (A. W: A. S. 1911, p. $1060-1066$ ). 
Ces dangers se sont trouvés aggravés par la crise contemporaine de déforestation issue do préoccupations fiscales qui poussent encore en France nombre de particuliers à réaliser hâtivement les vieux bois de lcurs forêts valorisécs par des épargnes séculaires. Pour obvier à la disette croissante de ces bois très raréfiés, des partisans aussi qualifiés qu'héroïques du maintien à toul prix de "l'aire boisée " de la France, n'ont pas hésité à projeter le reboisement d'étendues équivalentes à deux ou trois départements. Dans ce but, la loi votée précipitamment le $\mathrm{r}^{\text {er }}$ avril rgro par la Chambre, recourt plus que jamais à la nationalisation du sol ! C'est à proprement parler l'amorce d'une loi de "socialisation agraire " qui, au gré des circonstances, des idées... et des hommes, englobera fatalement un jour ou l'autre des territoires très variés, aussi bien en plaine qu'en montagne. Il est inutile d'insister sur les effets dépopulateurs qu'aurait une pareille législation, si clle était jamais sanctionnée pär le Sénat.

Les progrès contemporains de la dégradation du sol montagneux ne sont pas le fait exclusif de populations qui s'en évadent, et de moutons qui s'y raréfient. Les inestimables aptitudes naturelles et produits économiques du précieux laniger ont à tous les âges de la civilisation, et particulièrement dans les pays latins, poussé l'Etat à battre, avec lui, monnaie de ses terres pauvres. C'est en multipliant outre mesure le nombre de leurs moutons, autochtones ou transhumants, tous à des degrés divers, dévastateurs des sols accidentés, que la plupar des communes montagneuses peuvent encore riposter aux attaques incessantes of progressives du fisc. Telle est une des raisons essentielles de l'incapacité où se trouve aujourd'hui le pays, obstiné dans d'anciens errements, à restaurer ses montagnes.

La genèse contemporaine des "communes mortes " dans nos Alpes, et de celles auxquelles la nationalisation du sol y prépare une fin prochaine ; certaines tentatives actuelles de réduction des frais de la transhumance en vue de la faire ainsi survivre aux progrès de la dépécoration ovine ; le silence complaisamment gardé sur le rôle des spéculateurs pastoraux habitant le plus souvent la plaine qui, par. l'exploitation très fructueuse pour eux de cette transhumance, se trouvent être, au prix de pillages pastoraux avérés, les bailleurs de fonds des communes pauvres montagneuses, les garants en fin de compte de leurs charges fiscales, comptent ì des degrés divers dans un ensemble de contingences économiques ct socialos très actuelles qui ne scmblent pas avoir été beaucoup envisagées par le législateur de rgı.

Le problème se pose de plus en plus, dans nos hautes montagnes du Midi avant que, sous couleurs de restauration du sol, de reboisement, de spéculations techniques variées, voire de satisfactions à donner à des "intérêts personnels et immédiats ", on ne les ait entièremont dépeuplées, dé ménager au pays la possibilité et le droit d'y prendre, dans l'intérêt public, et sans y interrompre la vie sociale et pastorale, les mesures préventives que comporte la sauvegarde du sol, sans le dépeupler. Il faut chercher et appliquer la formule spéciale d'un Régime agraire (1) y inveslissant ce sol de privilèges fiscaux, successoraux et autres, susceptibles d'y atténuer dans une large mesure, pour celui qui l'occupe, aussi bien les "charges de l'altitude " inhérentes au milieu, que les restrictions de jouissance inhérentes à l'application des mesures de sauvegarde. Enfin, surtout en

(1) Legislation protectrice du sol montagneux en France (Journal des économistes. Avril 1911, p. 19-44 et La Houille Blanche. Nos de Juillet et d'Août 1911). haute montagne, le sol doit à tout prix rester aux mains de cclui qui le tient, qui s'y est adapté de longue date, ct quí seul peut le peupler.

Quel profit et surtout quelle gloire le pays tirerait-il jamais de la création de parcs nationaux alpins ou pyrénéens que, dans les circonstances actuelles, l'Etat ne pourrait constiluer qu'en pillant encore nos communaux montagneux ?

S'il convient aujourd'hui de rétribuer nos montagnards poux qu'ils continuent à habiter, à garder nos alpages et peut-être un jour à défendre certains d'entre eux (1), il faut savoir leur assurer des primes à l'enracinement el non, comme on l'a fait jusqu'ici, à l'expatriation.

\section{L.-A. Fabre.}

\section{L'AMÉNAGEMENT DES CHUTES D'EAU}

M. L.-A. Fabre a, dans l'article précédent, posé très nettement ce passionnant problème de la dépopulation des hautes montagnes, il en a montré les causes, dont une dés principales est la création coûteuse et mal comprise de parcs nationaux, les primes à l'expatriation, etc., il a indiqué les moyens que l'Etat pourrait employer pour en arrêter le mouvement.

Nous joignons à cette étude le rapport présenté par M. G. Charpenay à la séance du 5 juin 19 r 3 de la Chambre. de Commorce de Grenoble, en réponse aux attaques incessantes que tous, la presse quotidienne, le Parlement, les revues sportives, etc., dirigent contre les industriels de la houille blanche. Sans doute, ceux qu'ón a appelé des "Vandales " ont parfois déparé certaines vallées, construit de longs tuyaux inesthétiques, mais on ne met pas en parallèle les résultats inverses qu'ils ont obtenus. Ils repeuplent la montagne, reboisent les hauteurs, créent des lacs, dans beaucoup de villages morts, désertés, ils ont ramenés la richesse et la vie.

No serait-ce pas dans le développement des installations de la houille blanche que l'on trouvera 'une des solutions de ce problème, dont aucun bon Français ne peut se désintéresser?

\section{Charpenay donne lecture du rapport suivant :}

Messieurs, la Chambre de Commerce de Grenoble, à qui rien de ce qui touche aux intérêts économiques du Dauphiné ne saurait demeurer étranger ni mềme simplement indifférent et dont les membres appartiennent aux branches les plus importantes et les plus actives de l'industrie et du commerce de la région des Alpes, connaît certes mieux que quiconque l'essor formidable qu'a pris, au cours de ceś vingt dernières années notamment, l'exploitation des forces hydrauligues.

Apanage des pays montagneux, la houlle blanche, pour l'appeler d'un nom universellement adopté aujourd'hui, constitue par son abondance, sa pérennité et la multiplicité de ses avantages notre principale richesse locale : à tel point que, nul ne l'ignore et personne ne songerait à le nier, sa mise en valeur a véritablement transformé les conditions d'existence, si précaires jusqu'alors, de nos populations rurales, en même temps qu'elle a été ct reslera un élément puissant de prospérité, d'abord pour icéux qui l'utilisent, ensuile et par une conséquence inéluctable pour les départements de la France où clle est utilisée.

Au surplus, je n'ai pas la prétention de rien vous apprendre sur un sujet que l'éclatante manifestation du Coñ.

(1) La question de montrgnes depnis Gazanne. Les highlands de. France (La Montagne. Février 1912, p. 89-100). 
gròs de 1902, auquel la Chambre- de Commerce à cette epoque participa très largement, a déjà si complètement mis en lumière. Je n'appuierai pas davantage sur l'imporlance capitale que présente, pour notre Dauphiné surtout, l'aménagement des chutes d'eau, partout où elles existent, et sur le devoir qui incombe, dès lors, aux organismes à yui cst confiée la défense des intérêts régionaux, telle eı: premier lieu la Chambre de Commerce, de prendre toutes décisions el toutes mesures nécessaires à en faciliter l'exécution, à en assurer le bon fonctionnement el en favoriser La miltiplicalion.

Or, depuis quelques mois, un mouvement, dont le caractère tendancieux est de nature à préoccuper vivement ceux qui ont le souci de travailler à l'expansion de nos richesses hydrauliques, se dessine nettement, mouvement qui a pour but de faire échec à la captation, en vue des besoins industricls, des torrents de nos montagnes. Au nom de l'esthélique, et sous le prétexte quelque peu subtil que l'utilisation des caux des ruisseaux, en les détournant de leur lit naturd pour les cmmagasiner dans des tunnels ou des conduites fermées, nuit à la beaulé ou au charme du paysage, unlaines sociétés touristiques, le T. G. F., le Comité des siles of monuments, quélques Syndicats d'inilialive, voire des membres du Pailement n'appartenant pas, en général, a des régions de houille blanche, ont protesté, dans la presse ou à la Chambre, contre la création des chutes d'eau industriclles.

Lcur thèse est, en résumé, la suivante : la construction d'usines dans les sites pittoresques, où le tourisme aime à fréquenter, détruisant l'harmonie du paysage - d'aucuns sont allés jusqu'à dire que "les usines souillaient les valJées de leur présence ! " - nul doute que les visiteurs ne tarderonl pas à fuir ces régions, ce qui constituerait pour les populations, où leurs dépenses apportent du bien-être, un véritable désastre. Pour y obvier, un dépulé, à la tribune de la Chambre, n'a rien trouvé de mieux que de proposer le classement d'office des sites de nos montagnes dans la catégorie des biens nationaux!

Certes, je reconnais - é je le déplore tout le premier que, sinon les barrages et les battiments d'usines qui n'ont ricn de particulièrement inélégant, du moins les potcaux et les fils, véhicules de l'électricité engendréc par ces usines, quand ils occupent de longues distances sur les routes et chemins, ne sont point d'un effet harmonieux.

J'admets très volonticrs, cgalement, que te touriste soil un élément, non pas de bien grande richesse, toul au moins cependant d'une certaine prospérité pour les villes et villages des régions piltoresques qu'il parcourl la plupart du temps sans y séjourner.

Mais, je n'hésile pas à affurmer quion ne saurait élablir ancune comparaison entre les arantages que l'industrie, llune parl, et le lourisme, d'autre parl, apportenl avec cux aux populations de nos monlagnes. Si celui-ci donne à leur misère quelque soulagement, celui-la, par contre, conlribue puissamment à assurer leur existence même. Or, l'essenticl n'est-il pas, comme le disait un auleur ancien, de "virre d'abord", primo vivere? On se plaint depuis longlemps, el combien à juste litre; de l'exode, vers les villes, des travailleurs de la terre. C'est là un fait indéniable : la terre ne suffit pas dans notre région des Alpes, plus encore quailleturs, en raison de l'aridité du sol, dn morcellement infini de la proprićté, à faire vivre l'habitant. qui, dès lors, se voit contraint d'aller demander aux grands centres une rémunération de son travail plus adéquate atux nécessités de l'existence.
Eh bien I ce que la terre refusait autrefois à l'indigène de l'Oisans, de la Maurienne, par exemple, regions si particulièrement dures et tourmentées, les nombreux torrents qui les sillonnent le lui domnent aujourd'hui; grâce aux nombreuses usines échelonnées le long du cours de la Romanche et de l'Arc et nées de ces torrents, non seulement les populations ne s'exilent plus, mais lo nombre des habitants de nos hautes vallées s'accroîl sans cesse. Là où, il $y$ a vingt ans à peine, on ne rencontrait que quelques maisons éparses et d'aspect misérable, s'élèvent aujourd'hui de véritables et coquettes petiles villes : je n'en veux citer pour exemple que Rioupéroux, Livet, Saint-Michelde-Maurienne, La Praz, etc...

Cette bienfaisante révolution n'est cerles pas due au tourisme, mais elle est bien réellement la conséquence du puissant essor industriel qu'a provoqué l'asservissement des torrents, l'aménagement des chutes d'eau !

Aussi bien, Messieurs, je crois en avoir assez dil sur une question que votre situation et volre expérience personnelles vons ont rendue si familière.

Je n'insisterai donc pas davantage. Je vous demanderai simplement de vouloir bien, comme l'ont déjà fait notamment nos collègues de la Ghambre de Commerce de Gap, dans leur séance du 29 janvier gr 3 à l'ocoasion de la chute du Guil, comme le fail chaque fois qu'il sagit de prendre la défense des intérêts d'une industrie qui est, je lo répète, la principale richesse de nos Alpes, la Chambre syndicale des Forces hydrauliques, je vous demanderai, dis-je, de vouloir bien donner aux considérations qui précèdent la sanction de votre haute autorité en adoplant le vou ciaprès que l'honeur de vous soumeltre :

\section{"La Chambre de Commerce de Grenoble,}

"Tout en reconnaissant qu'il convient de respecter, dans la plus largo mesure compalible avec les intérêts économiques du pays, les beautés naturelles des sites el paysages dans les régions fréquentées pendant la belle saison par lo tourisme :

" Mais considérant que la création des chutes d'eau el r'aménagement des usines el des installations industrielles, qui en sont la conséquence nécessaire, constiluent pour les régions montagneuses un élément de bien-être et de richesse de premier ordre;

"Que, notamment les industries clablies grâce a l'ulilisalion des forces hydrauliques dans des régions ou l'aridilé du sol, le morcellement de la propriélé, l'inclémence des saisons, ne permettaient pas juscju'alors aux populations d'y vivre, ont, partout où dles sont installés aujourd'hui. enrayé complètement l'exode des habilants et contribuent meme à accroitre, dans de sensibles proportions, les populations des villages alpestres auxquelles lo travail à l'usine fournil des solaires assurant largement low existence of celle de leur famille;

"Que les ressources apportées à ces populations par le lourisme ne sauraient aucunement, en raison de linstabilité el de Ja courte durée de la période touristique, étre comparés à celles iquine industrie installée et fonctionnant en permanence peridant toute lannée sur leur terriloire, leur procure;

"Qu'enfin l'industric des forces hydrauliques ne conslitue pas miquement un elément de vitalité et de prospérilé pour les seules communes oì rlle s'exploite réllement, mais qu'elle exerce encore une influence consiforable sur la piospérilé générale du pays tout entrer; en vertu de ce 
principe que la "richesse d'une nation est fonclion de la " richesse de lensemble des pays qui composent celle "nation " .

\section{"Pour tous ces molifs,}

"La Chambre de Commerce de Crenoble, eslimant que l'intervention du Ministère des Beaux-Arts dans la question d'ulilisalion des forces hydrauliques ne pourrail que compliquer inutilement, sinon même entraver l'exécution des projets de créalion de chutes d'eau déjà soumis, en l'état, aux Ministères des Travaux publics, de l'Agricullure el des Finances, et donl la solution n'est trop souvent si lente à intervenir, en raison de la multiplicité des formalités administralives dont leur approbation est entourée.

"Emel à l'unanimité de ses membres l'avis que l'intervenlion du Minislre des Beaux-Arts est absolument inutile et qu'elle ne pourrait être que préjudiciable au développement de l'induslrie de la houille blanche, une des richesses nalurelles les plus considérables d'une grande partie de la France;

"Elle proteste énergiquement contre la campagne ayant pour bul ou pour résultal de faire échouer ou d'enrayer les projels nés ou à nathe en vue de l'ulilisation des ressources hydrauliques des Alpes el protesle également contre le classement des sites comme biens nationaux, ce classemenl ne pouvant avoir pour effet que de paralyser l'essor d'industries nouvelles, auxquelles le Dauphiné doit, en grande partie, son magnifique développement économique et qui, en donnant anx populalions déshérilées de nos monlagnes la possibilite de vivre désormais sur lear territoire, contribuent si puissamment à metlre fin au paupérisme et à enrayer l'émigralion.

"Comme conséquence, la Chambre demande à $M$. le Préfet el à MM. les Représentants du Départernent d'intervenir dans ce sens axprès des Adminislralions consultées pour hâler les formalilés présenles en ce qui conceme les projels en voie d'approbalion. "

La Chambre de Commerce de Grenoble a approuvé les termes et les condusions de ce rapport.

\section{LE NITRURE D'ALUMINIUM}

\section{Les Brevets français relatifs à cette fabrication}

Quels sont les brexets frangais qui sont, ou vonl etre, appliqués dans la fameuse industric naissante du nitrure d'aluminium, el quelle est la valeur réelle de ces brevels ? Celle question nous a ćté souvent posée, et pour l'éludier, sinon pour la résoudre, nous avons abordé l'élude de la très voluminense liltérature que forme déjà l'ensemble des brevels français publićs jusqu à ce jour rolativement à la fabricalion du nitrure d'aluminium. Nous litisserons de côlé, pour le momenl du moins, les brevels relatifs a la decomposition de ce nitrurc.

Non seulement celle litlérature très spéciale est volumineuse : mais elle est terriblement indigeste I Le slyle de presque tous ces brevels n'a que de loinlains rapports avec le bon français ordinaire. Il semble que tous les inventeurs, même frangais, commencent par penser el rédiger on allemand, pour traduirc ensuite et mot à mot en francais. D'autre part, un certain nombre de brevets semblent avoir ćté déposés en vue de gêner ou dérouter les concurrents, et lícrivent avec un grand luxe de délails oiseux des disposilifs imaginés de toute pièce a priori et sans aucune intention de les réaliser jamais. Enfin, et surtout, de mème quo, lorsqu'on visite une usine, si on n'èst pas tout à fait spécialiste, on n'aperçoil pas les détails les plus nouveaux et les plus importants, de même, si on n'est pas de la partie, on ne distingue pas facilement dans les brevets relatifs à la fabricalion des nitrures, les particularités réellement importantes. Or, qui donc peul se dire vraiment " de la partie $n$, en dehors des deux très petits groupes de techniciens rivaux ef concurrents qui ponrsuivent actuellement, en grand secret, dans deux vallées voisines de la Savoie, la mise à point de la nouvelle fabrication ?

Nous n'avons donc d'autre prétention, dans l'exposé quil va suivre, que de donner un apercu de la complexité du problème. Assurément la question ne restera pas toujours aussi nuageuse ; bientòl poul-être, c'est-à-dire dans un an ou deux, on saura quels sont les brevels réellement utilisés ou ulilisables.

On peut compter unc trentaine de brevets français publiés jusqu'à ce jour pour la fabrication du nitrure d'aluminium. En outre, une douzaine de brevets français ont été déposés, mais ne sont pas encore publiés, et, naturellement, ce sont les plus importanls ! Dès lors l'examen des brevets déjà publiés ne peul donner une idée exacte de la nouvelle industrie, actuellement en pleine évolution.

Nous préscnterons donc simplement une liste des principales particularités brevetécs jusqu'à ce jour,. $1^{\mathrm{or}}$ juillet 1913. Les letures majuscules désigneront les particularités. qui nous paraissent lombées dans le domaine public en France ; les lettres minuscules désigneront les particularilés. qui nous paraissonl au contraire garantics par des brevels particuliers achuellement encore en vigueur.

A. - C'est en 1862 que le nilirue d'aluminium a élé découverl, el ses propriélés chimiques essentielles ont été décrites on 1876 , mais il s'agissait de recherehes de laboraloire. La fabrication industrielle de ce nouveau corps est une première parlicularilé imporlante qui a été brevetée par Willson (brevet anglais 2 I 755 du ${ }^{5} 5$ novembre 1895 ). Le brevel français correspondant n'ayant pas été demandé, celte particularité est dans le domaine public en France depuis lc 16 novembre 1896 .

B. - Fabrication industriclle par chauffage, en présence d'azote, d'un mélange d'alumine el de charbon. Brevel Willson du $x 5$ nov. 1895 . Briegleb et Geuther, en 1862 , el Mallel, en 1876 , avaient oblemu le nilrure d'aluminium en partant de l'aluminium mélal. Meme observation que pour $A$.

C. - T.e chaulfige est effectué "dans un four électrique approprié ". Brevet anglais de Willson de $1 \dot{8}_{95} 5$. Même observation que pour $A$.

D. - Ce four électrique est à arc el à résislance, la résistance étant conslituée par la matière à traiter. Ie texle du brevet Willson, de 1895 , dit : a Dans un four électrique approprié, préférablement dans un four à arc ", et les trois figures $I, 2$ et 3 du brevet représentent bien, en effet, une électode mobile verlicale, placée à une certaine dislance des malières à traiter, donc engendrant un are entre elle-même et ces malières. Mais ces mêmes figures montrent que s'il se prodüt un are entre l'électrode mobile et les malières à traiter, par contre au-delà de cet arc et pour gagner la sole conductrice du four, le courant tra- 